\section{Malestar emocional en pacientes de cuidados paliativos: desafíos y oportunidades}

\section{Emotional malaise in palliative care patients: challenges and opportunities}

\section{Señor Editor,}

Actualmente en Chile el cáncer es una patología que afecta a un porcentaje importante de la población y representa una de las principales causas de muerte en el país. Uno de los aspectos centrales en el abordaje del cáncer es la consideración de los pacientes que se encuentran en etapa avanzada de su enfermedad, para quienes no existe un tratamiento curativo de la misma $y$, por ende, se les recomienda el ingreso a cuidados paliativos.

Desde el año 2005 Chile cuenta con una Guía de Alivio del Dolor por Cáncer Avanzado y Cuidados Paliativos ${ }^{1}$, la cual da lineamientos a los equipos clínicos respecto a la evaluación y alivio del dolor de los pacientes en etapas avanzadas de su enfermedad, apuntando al alivio del sufrimiento físico y emocional de los pacientes y sus familias, considerando un abordaje de cuidados humanizados en salud. En el contexto de la humanización de la salud, en la que los pacientes ocupan un lugar central en la atención y los cuidados, las necesidades psicosociales de los mismos son especialmente relevantes. Así lo reflejan las recomendaciones de la National Comprehensive Cancer Network [NCCN] sobre la necesidad de un enfoque integral en la atención del paciente oncológico ${ }^{2}$. Si bien la Guía de Alivio del Dolor por Cáncer Avanzado y Cuidados Paliativos releva estas temáticas, ésta no cuenta con lineamientos específicos para el abordaje de los problemas emocionales que presentan los pacientes y sus familias en el contexto de los cuidados paliativos ${ }^{3}$.

La enfermedad oncológica avanzada conlleva una variedad única de problemas psicosociales que con frecuencia generan malestar emocional, un concepto propuesto por la NCCN y que se define como "una experiencia emocional desagradable y multifactorial de naturaleza psicológica, social y/o espiritual que puede interferir en la capacidad de afrontar de forma efectiva el cáncer, los síntomas físicos asociados y el tratamiento" 4 . El malestar emocional se extendería a lo largo de un continuo que va de sentimientos normales de vulnerabilidad, tristeza y miedo, hasta problemas más complejos como la depresión, ansiedad y trastornos de pánico. El creciente interés en el malestar emocional y la importancia de su abordaje ha llevado a que se le considere como el $6^{\circ}$ signo vital después de la temperatura, la respiración, la frecuencia cardiaca, la presión arterial y el dolor como indicadores de salud y bienestar del paciente ${ }^{4}$. Así mismo, ha sido incluido como un mínimo en la acreditación de los programas de cáncer hospitalarios, convirtiéndose en un elemento clave del cuidado integral de los pacientes oncológicos.

Si bien, todos los pacientes presentan algún grado de malestar emocional, en enfermedad avanzada la prevalencia de malestar emocional significativo alcanza una cifra cercana a $80 \%{ }^{5}$. Desafortunadamente, a pesar de la alta prevalencia, pocos pacientes con necesidad de apoyo emocional profesional recibe tratamiento. No identificar ni abordar el malestar emocional puede repercutir en problemas de adherencia terapéutica, dificultar los procesos de toma de decisiones, disminuir la calidad de vida y aumentar el gasto al realizarse más visitas médicas y a los servicios de urgencia, así como prolongar las estadías hospitalarias.

En Chile, la realidad psicosocial de los pacientes oncológicos en cuidados paliativos es un ámbito de estudio que aún presenta muchas aristas por explorar. Carecemos de información relativa a su malestar emocional, no contamos con instrumentos específicos que faciliten su detección, ni con guías que orienten a los profesionales de la salud implicados en su cuidado en cómo abordar las necesidades emocionales de los pacientes y sus familias. Dada la relevancia que en este minuto está tomando el cuidado del paciente oncológico a través del Plan Nacional del Cáncer, que entre sus objetivos cuenta con garantizar el acceso a los Cuidados Paliativos, resulta esencial abordar la problemática psicosocial de estos pacientes con el objetivo final de garantizar un cuidado integral y de calidad. Hoy tenemos la oportunidad de relevar esta temática y generar acciones que se orienten a evaluar y aliviar el malestar emocional de los pacientes oncológicos de manera oportuna.

\section{Margarita Bernales ${ }^{1}$, Alejandra Chandía ${ }^{2}$, María Jesús San Martín \\ ${ }^{1} \mathrm{PhD}$, Escuela de Enfermería, Pontificia Universidad Católica de Chile. Santiago, Chile. \\ ${ }^{2} \mathrm{MSc}$, Centro del Cáncer, Hospital Clínico Pontificia Universidad Católica de Chile. Santiago, Chile. ${ }^{3} \mathrm{MSc}$, Escuela de Psicología, Pontificia Universidad Católica de Chile. Santiago, Chile.}

\section{Referencias}

1. Ministerio de Salud. Guía Clínica AUGE Alivio del Dolor por cáncer avanzado y Cuidados Paliativos. Serie Guías Clínicas MINSAL. Santiago de Chile, 2011.

2. National Comprehensive Cancer Network (NCCN). Practice guidelines for the management of psychosocial distress. Oncology 1999; 13: 113-47.

3. Tuca A, Schroder M, Novellas A. Cuidados paliativos en oncología. Anuario de Psicología, Facultad de Psicologia. Universitat de Barcelona 1998; 29 (4): 35-53. 
4. Bultz B, Holland J. Emotional Distress in patients with cancer: The six vital sign. Community Oncol 2006; 3: 311-34.

5. Díaz-Frutos D, Baca-García E, García-Foncillas J, LópezCastroman J. Predictors of psychological distress in advanced cancer patients under palliative treatments. Eur J Cancer Care (Engl) 2016; 25 (4): 608-15.
Correspondencia a: Margarita Bernales

Vicuña Mackenna 4860. Campus San Joaquín. Escuela de Enfermería, Of. 115. Santiago, Chile.

mmbernal@uc.cl 